\title{
CARROT-AND-STICK PROCEDURE WITHOUT CARROTS: VICARIOUS PUNISHMENT PROMPTS AND SYSTEM TRANSPARENCY IN E-LEARNING GROUPS
}

\author{
Filipa Stoyanova and Nicole C. Krämer \\ University of Duisburg-Essen, Forsthausweg 2, 47048 Duisburg, Germany
}

\begin{abstract}
A common cause for low satisfaction with group work in online courses is that teammates might be inactive and need to be prompted to take up work. Here, we specifically focus on the question of whether it is beneficial when the visualization of inactivity by the system is accompanied by negative consequences. In a field experiment in an online learning course we combined repeated questionnaires and behavioral data to examine whether vicarious punishment and transparency improve users' behavior (e.g. participation) and subjective perception (e.g. teamwork satisfaction). In a 2(x2) between-subjects design ( $N=81$, conducted over the course of 4 weeks), an abstract system signaled inactivity, whereby inactive teammates (confederates) were publicly addressed by name or not (vicarious punishment/no vicarious punishment condition). Additionally, to gain insights into how much needs to be known about the system's functioning, in the vicarious punishment condition participants were informed about the system's functionality in more/less detail, i.e. the system's transparency was varied (high/low transparency condition). Participation equality predicted teamwork satisfaction. Vicarious punishment led to more constructive prompts, but less observability of others, i.e. the ability to estimate others' contribution, which was even lower in the transparency condition. However, transparency revealed participants to be more afraid and a negative correlation with number of contributions emerged.
\end{abstract}

\section{KEYWORDS}

Vicarious Punishment, Transparency, Prompting, E-Learning, Online Collaboration, CSCL

\section{INTRODUCTION}

Group work is hailed as one possibility in online courses to deepen learn processes and to keep learners motivated (Erdmann et al., 2017). However, this does not always increase motivation as free-riding of single group members is a common problem (Strauß, Rummel, Stoyanova, \& Krämer, 2018). In order to avoid free riding, teammates have to usually prompt the free-riding members themselves, which might be demanding when done as personal remarks. Alternatively, automated support and warning systems could be employed in online environments. This is especially important in e-learning teams, where ignoring instead of signaling problems might be an indirect reward for teammates who are responsible for the problematic behaviors, but barely experience consequences. On the contrary, punishing those in charge might be rewarding for other, actively participating teammates, as they are not subjected to the same form of punishment. In this line, not punishing free-riders is an indirect punishment for active contributors. Since adding motivational issues for effective behaviors is not always effective (Kyewski \& Krämer, 2018), addressing instead of ignoring problematic behaviors might help to handle them in computer supported collaborative learning (CSCL). We therefore ask: Is vicarious punishment an underestimated solution in cases of inactivity in e-learning groups? How could the process of automated prompting and the delivery system be optimized?

Little research to date has focused on vicarious punishment in online learning groups. In classic, offline settings in the realm of working psychology as well as in traditional classroom settings, it was shown to increase the opposite of punished behavior, e.g. work contribution, but not participants' satisfaction (Schnake, 1987). However, vicarious punishment in online settings delivered by a system was not investigated so far (Malouff, Thorsteinsson, Schutte, \& Rooke, 2009), even though it has the potential to be perceived differently and capitalize the effects already shown in offline settings. 
Prior research has already indicated some benefits of intelligent support in CSCL. In an online experiment, prompting messages to increase teammates' activity in online learning groups have already been shown to improve users' perception of message persuasiveness and sender impression when delivered by an abstract system instead of other teammates (Stoyanova \& Krämer, 2019).

When the system is a relevant interaction partner which provides punishments, it is also important what the user assumes about the functioning of the system. This has been investigated as transparency and showed contradicting effects, e.g. in the realm of recommending systems (Sinha \& Swearingen, 2002). Thus, transparency can potentially influence whether the user perceives the system to be able to announce feedback.

To date transparency and vicarious punishment have neither been investigated combined in an e-learning setting nor in the long term. Therefore, the aim of the present study is the comprehensive investigation of the effects of vicarious punishment and transparency on e-learning small groups. Especially the combined analysis of objective and subjective data in a real online course can help to understand how to prevent inactivity and how to optimize the prompts delivered by the system. To the best of our knowledge, there are no results in the literature regarding how these factors affect computer supported collaborative learning, the perception of prompting messages and of the prompts delivery system.

\section{THEORETICAL BACKGROUND}

\subsection{Vicarious Punishment}

When punishing unacceptable behaviors, opposites are rewarded by the simple absence of a punishment for them. "(...) When we fail or are unable to punish a difficult employee, we are, in effect, punishing those employees who have not engaged in deviant behaviors and rewarding those individuals who have" (McCallister in Schnake, 1987, p. 379). According to the concept of a vicarious punishment the demonstration of negative consequences for inactivity may be an effective warning with rewarding effects among the remaining (active) teammates. Vicarious punishment has mostly been studied in terms of classic offline laboratory experiments, as simulation of work related or classroom settings or with younger participants. In the meta-analysis with 21 studies, observing others getting punished for a certain behavior led to lower levels of the same behavior by the observers. According to Malouff's characteristics of the analyzed studies, the present study could be categorized as follows, displaying the differences and gaps of previous literature in this realm. The key difference is with regard to the observation of a punishment model. The present study takes place in an online learning environment, the punishment of the model is neither filmed nor live, differing from all the studies analyzed by Malouff et al. (2009). In our study, the model is another confederate teammate from the online course and the observation of its "punishment", i.e. the publicly criticizing by name, is in real time online. "A public reprimand for cheating (...) or publicly criticizing" (Malouff et al., 2009, p. 274) as severe punishments are similar to highlighting of inactive teammates in teamwork, where the punishment type corresponds to a solely positive one, i.e. the addition of an undesirable stimulus.

In the case of e-learning, both detrimental and beneficial effects on students' behavior and perception of the system can be assumed. Beneficial effects could arise, such as motivation to avoid the black sheep role, increased satisfaction with group work due to perception of fairness and justice or even increases of regulative interactions as an imitation of the regulation acts of the system. On the other hand, participants decide to imitate the inactive teammates, in case negative consequences are missing (O'Fallon \& Butterfield, 2012). Hence, in the present field study, prompts should directly address an inactive teammate (an artificial confederate) in order to investigate, if a system is allowed to intervene in a more severe way. Would participants rather focus on negative behaviors, imitate or quit them due to automated mediation? Further attributes of the intervening system, such as transparency, could offer an answer to these questions and help to find automated means to increase satisfaction and prevent drop-out.

\subsection{Transparency}

Detailed information about the system's functionality will be addressed as system transparency. In research literature it has already been shown as beneficial in the specific case of recommender systems - by increasing confidence and liking (Sinha \& Swearingen, 2002). However, another study regarding a financial advisory 
system does not show positive effects of revealing the system's functionality, which is interpreted as an effect of disappointed expectations (Nussbaumer \& Matter, 2011). Further, in the realm of human-robot interaction, within a group task, when the delivery robot was more transparent, less blame and credit was attributed to other teammates, however, transparency did not affect credit or blame to the robot (Kim \& Hinds, 2006). Another study regarding trust and transparency recommended balanced interface transparency - neither too little, nor too much (Kizilcec, 2016). Individuals whose expectations were violated (by receiving a lower grade than expected) trusted the peer assessment system less, unless the grading algorithm was made more transparent. However, too much information eroded this trust and attitudes did not vary with transparency, when expectations were met.

Certain characteristics could replace system's proficiency, signal expertise and credibility, and finally enhance system's legitimacy to highlight inactive teammates. It was already demonstrated that message perception, sender impression and internal causal attribution were facilitated when prompts were sent by a system instead of an average or low proficient fellow student (Stoyanova \& Krämer, 2019).

Based on the reported theories and findings we hypothesize:

H1: The higher equality of user participation increases satisfaction with group work.

H2: Vicarious punishment has a positive impact on the subjective user perception of inactive teammates (2.1), group awareness (2.2) and satisfaction with group work (2.3), as well as on the objective user behavior such as login frequency (2.4), contribution quantity in forum (2.5) and wiki (2.6), and equality of participation (2.7). H3: Vicarious punishment has a negative impact on the subjective user perception of the system (3.1) and the prompt messages (3.2).

Based on the contradicting results regarding the system transparency, in an explorative approach its effects will be investigated by formulating the research questions:

RQ1: What influence does transparency have on the subjective user perception of the system (1.1), and the prompt messages (1.2), group awareness (1.3) and satisfaction with teamwork (1.4)?

RQ2: What influence does transparency have on the objective user behavior?

E-learning is mostly conducted in the long term, i.e. within a longer period of time instead of a single lesson. Interacting with the prompting system repeatedly can influence the perception of the system in various ways. Potential repetition effects could weaken or strengthen the effects of vicarious punishment and transparency. Thus, the development over time is of additional interest:

RQ3: Is there a difference between first and second group task regarding stress, fear, tendency to imitate inactive teammates, and equality of participation depending on vicarious punishment and transparency?

Additionally, based on inconsistent results within research on transparency and on the research gap regarding vicarious punishment in e-learning groups, it is necessary and important to examine the influence of subjective variables like system and prompts' perception (objectivity, faultiness, comprehensiveness etc.), as well as perceived in-group variables (stress, conflict, trust) on satisfaction, fear and, lastly but importantly, on behavioral outcomes (contribution amount, login frequency). Thus, we stated the research questions:

RQ4.1: What relevant factors influence users' objective behavior? Is there a correlation between fear, login frequency and contribution?

RQ4.2: What relevant factors influence users' subjective in-group experiences? Is there a correlation between the perception of the prompts with the perception of conflict and in-group trust?

RQ4.3: What relevant factors influence users' subjective perception of satisfaction and fear? Is there a correlation between satisfaction, the tendency to imitate inactivity and fear?

Further, it would be interesting to examine whether vicarious punishment could predict the contribution amount, as it influenced productivity in offline experiments (Malouff et al., 2009), and whether it depends on the perceived fear. The fear to get publicly criticized might increase the motivation to log in more frequently or, vice versa, the fear to get criticized might lead to rare login actions to avoid the criticizing. On the other hand, the more frequent login might result in more contributions in forum and wiki. These thoughts lead to the following research question:

RQ5: Will vicarious punishment increase the fear and login frequency, resulting in more total contribution? 


\section{METHOD}

The study was conducted at a German university in the winter semester of 2019/2020 within an online course addressing the psychological basics of computer mediated communication. The course was implemented in the online learning environment Moodle and comprised six topic blocks combined with group tasks. The course materials included a video as a topic introduction as well as basic literature and short optional quizzes. Each group task had to be completed in small groups of three to five students within 14 days. The course participants were randomly assigned to groups at the beginning of each topic block, so that always new teammate constellations were assured. This way habituation effects in the groups were avoided and new coordination as well as exchange processes were stimulated. The teams had their own group forum and a wiki as a text production tool in order to coordinate and develop the group contribution.

\subsection{Field Experimental Design and Procedure}

The present study was placed in the first half of the course, over a period of two topic blocks (4 weeks in total). The field study employed a 2(x2) experimental between-subjects design. Vicarious punishment and system transparency were varied as independent variables. The design is not fully crossed as system transparency was only varied in the vicarious punishment conditions.

A group awareness tool visualized the quantity of forum and wiki contributions within the team in a bar graph. The system signaled when a teammate has not been participating actively by displaying prompt messages on a group level regarding the potential for improvement and coordination hints. In the vicarious punishment condition, inactive teammates (confederates) were addressed by name. A pre-test was used to evaluate potential formulations $\left(N_{\text {pretest }}=40\right)$ and choose neither unfavorable, nor extremely severe ones.

In order to apply vicarious punishment, profiles of confederate course participants were generated, left inactive and assigned to each group. From the participants' point of view, the confederate profiles did not differ from the others. Teamwork was assessed as passed or failed on a group level and did not influence the individual course grade, so that the confederates' inactivity was not a disadvantage for the real course participants. Severe vicarious punishment was varied by publicly criticizing an inactive confederate teammate (vicarious punishment condition) (Figure 1). Criticizing was placed in the prompting message from the system in the group awareness tool. The prompting message occurred at the fourth day and was kept visible until the end of the task in a team tool on the platform.
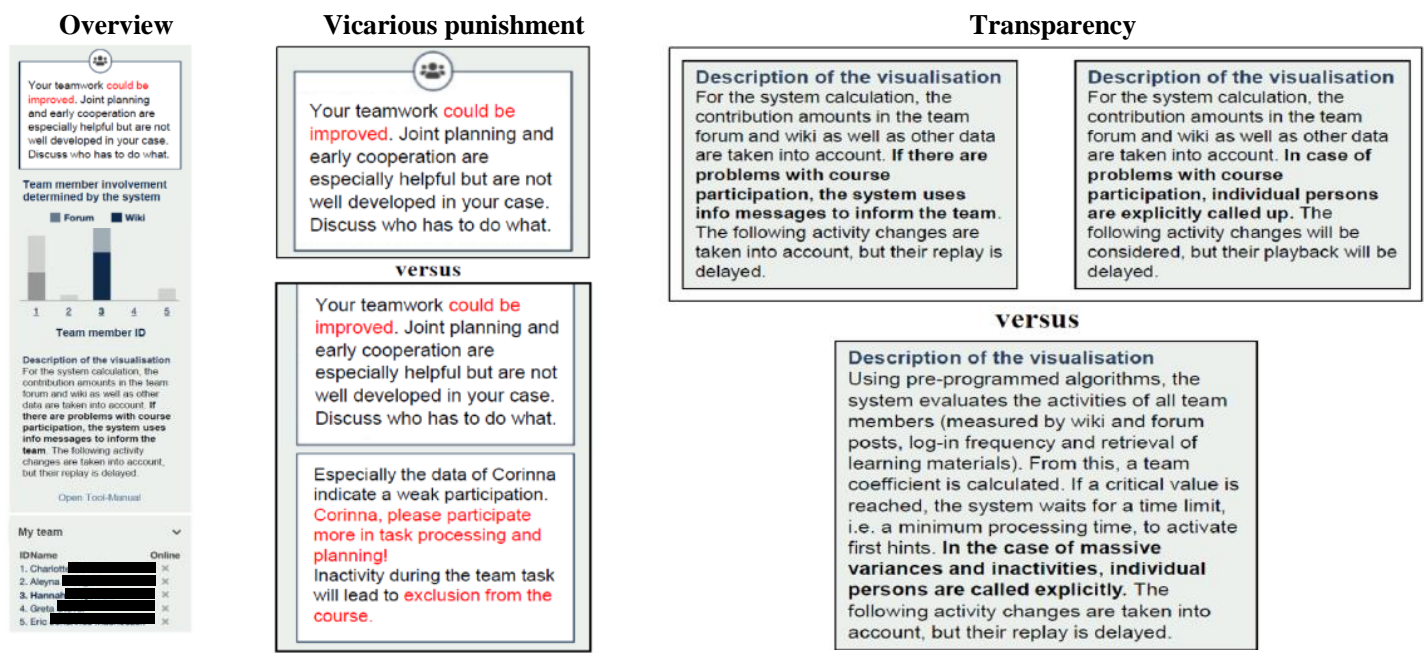

Figure 1. Left to right: Group awareness tool overview, varying vicarious punishment and transparency in the conditions

Additionally, the system transparency was varied by informing the participants in advance more or less detailed about the system's functionality (high vs. low transparency condition). At the beginning of the first topic block, a more or less detailed briefing about the functionality of the system was sent per e-mail and applied on the platform as an obligatory pop-up text and as a visible short version within the tool (Figure 1). 
In the high transparency condition it included a list of all possible sources for the inactivity calculation and an explanation of the thresholds before activity changes are displayed. The transparency manipulation was only applied within the vicarious punishment condition.

The independence of the accompanying research and the course was clearly communicated. Written informed consent was obtained by the course participants, who agreed to participate in the accompanying research voluntary. Participation could have been cancelled any time without justification or consequences for the further running course.

With their consent to attend, the participants agreed to the storage of their objective data like Moodle activities and subjective data from the online surveys. These were due before the start of the course and after each two-week teamwork. The course activities were stored as log files. As an incentive for the continuous participation in the accompanying research, participants who filled all surveys were awarded a postpaid incentive of 10 Euro and 2x50 Euro were raffled. The procedure of the study was approved by the ethics committee of the university.

\subsection{Sample}

In total, 107 university students registered at the beginning of the course and 97 retained after the first week. $81(82.5 \%)$ of them agreed to participate in the accompanying research. For the first topic block with a team task, a total of 72 participants were randomly assigned to one of the three conditions $(n=24)$ and to teams, including four teammates and an inactive confederate. This way, six teams per condition were assigned. Due to high course drop-out rates $(31 \%)$, a total of 67 students retained in the second topic block of the course, of whom 57 study participants were assigned to the experimental conditions ( $n_{\text {no vicarious pun. }}=19, n_{\text {vicarious pun. }}=21$, $n$ vicarious pun. transparent $=17$ ).

The $\log$ file data of all 81 study participants were stored $(n \log$ files $=81)$. Most of them completed the pre-survey before teamwork $\left(n_{\text {pre-survey }}=80,51\right.$ females, $\left.M_{\text {age pre-survey }}=23.47, S D=3.11\right)$. Additionally, the same survey needed to be completed twice - after each teamwork block every two weeks, so that irregular participation was identified $\left(n_{\text {survey-t1 }}=52, n_{\text {survey-t2 }}=44\right)$ and in sum 42 participants completed all three surveys $\left(N=42,28\right.$ females; $\left.n_{\text {no vicarious pun. }}=15, n_{\text {vicarious pun. }}=14, n_{\text {vicarious pun. transparent }}=13\right)$. Data from drop-out students was used partly, depending on the short-survey they completed.

All participants were students, mostly of the Human sciences (42\%), Engineering sciences (20\%), Communication and Media (15\%) or Economics (14\%). They identified flexibility $(M=4.55, S D=0.75,1-5)$ and interest for the topic $(M=4.13, S D=.90,1-5)$ as the most important reasons to choose this course. On average, participants had a neutral attitude towards online teamwork participation $(M=3.22, S D=0.60,1-5)$ and neither negative nor positive prior experiences with group work $(M=48.91, S D=24.20,1-100)$.

\subsection{Measures}

The systematic field setting guaranteed access to objective behavioral data and subjective data (assessed in online surveys after each group task). Students' behaviors on the platform (click behavior, frequency of logins, quantity of contributions in forum and wiki, and the level of equality of participation) were logged as objective measures. The system could log the quantity, but not the quality of the behaviors. There is no information about how students processed materials or proceeded after accessing the platform and the materials. Log files were stored per topic, ordered by time and assigned to the platform ID of each participant, which were also transmitted to the online surveys. This method provided us the opportunity to relate self-reported data and log files.

In the first, "pre-survey" that was administered at the beginning of the four weeks, students were asked about socio-demographic parameters (age, sex, study program). Course related characteristics and their relevance for choosing this course were asked employing a 5-point Likert scale (1 to $5=$ strongly agree, 9 items, e.g. "as a personal challenge"). Additionally, we assessed topic knowledge and enjoyment of group work on a 5-point Likert scale ( 1 to $5=\mathrm{a}$ lot), as well as number of participation in Moodle courses and in online courses with group work (both input fields), previous experiences with online courses and with Moodle (slider 1 to $100=$ highly positive). We additionally measured personality traits with the short version of the big-five, goal orientation (other-approach, other-avoidance), self-efficacy, social comparison, innovativeness, social 
comparison orientation, schadenfreude, resignation and competitive learning attitude, which are not relevant for the analyses presented here.

After each of the two group tasks, a questionnaire with 39 items was employed (for an overview of detailed example items, reliability and descriptive statistics of all dependent variables, see Table 1). Primarily, the frequency of noticed system prompts, a recall of their content and the consequences of inactivity were asked as a manipulation check. Input fields were applied for these and for a final item regarding personal reactions and means in case of inactivity to foster collaboration. The rest of the questionnaire employed a 5-point Likert scale ( 1 to $5=$ strongly agree). We assessed the system perception and prompt message perception. For the factor analysis of prompt perception, means of both measurement times per item were applied. The recommended three-factorial solution was applied with objective, motivating, hostile. Regarding collaboration, we assessed the stressfulness of the last teamwork, satisfaction with group work, in-group trust, group conflict, stressful coordination and perception of inactive team members. We also measured group awareness, as well as an additional item on potentially varying task characteristics - degree of freedom during task processing. Questionnaires are available in an open science platform (see link* below the references).

Table 1. Measures

\begin{tabular}{|c|c|c|c|c|c|}
\hline & Variable & Item example & $\begin{array}{l}\text { s } \\
\text { al }\end{array}$ & $\begin{array}{r}\mathrm{T} \\
M_{t 1}\end{array}$ & $\begin{array}{l}S D_{t 1} \\
\text { tal }\end{array}$ \\
\hline \multirow{4}{*}{ స్ } & Fact-based character & Participation graph and prompts corresponded to reality & 1 & 3.78 & 1.05 \\
\hline & Emphasis calming & I'm reassured that action is taken against inactive members & 1 & 3.62 & 1.26 \\
\hline & Commiseration & I feel pity when a person is flagged as inactive & 1 & 2.42 & 1.23 \\
\hline & Fear of emphasis & I'm afraid I might get emphasized soon too & 1 & 2.33 & 1.04 \\
\hline \multirow{4}{*}{ 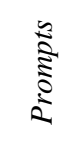 } & Noticed prompts & How often did you notice system hints system last 14 days & 1 & 5.09 & 3.80 \\
\hline & Objective & faulty(r), objective, comprehensible, appropriate & $4 \quad .90$ & 3.83 & 0.69 \\
\hline & Motivating & constructive, helpful, motivating, reassuring & 4.80 & 3.28 & 0.70 \\
\hline & Hostile & hostile, humiliating, threatening & 3.79 & 2.19 & 0.83 \\
\hline \multirow{5}{*}{ 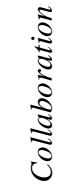 } & Stress & How stressful was your teamwork in the last 14 days & $2 \quad .59$ & 3.17 & 1.15 \\
\hline & Satisfaction & Overall I'm satisfied with teamwork in the last 14 days & 2.87 & 2.76 & 1.09 \\
\hline & In-group trust & I trusted my teammates to work on our task & 1 & 3.02 & 1.10 \\
\hline & Conflict & There was much tension among the members of our team & 1 & 2.10 & 0.98 \\
\hline & Coordination stress & Task division and workflows were not clear and precise & 1 & 3.25 & 1.23 \\
\hline \multirow{4}{*}{ 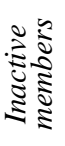 } & Existence and presence & I was very aware that there were inactive team members & 1 & 4.24 & 1.12 \\
\hline & Disliked peers & Such teammates are unpleasant co-workers & 1 & 3.73 & 1.17 \\
\hline & Disliked compensation & It was annoying to compensate for what others didn't do & 1 & 3.31 & 1.19 \\
\hline & Imitation tendency & When I see others' inactivity, I feel like quitting work too & 1 & 2.57 & 1.25 \\
\hline \multirow{5}{*}{ 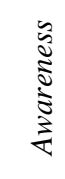 } & Hints used to optimize & We as a team used the prompts to change our collaboration & 1 & 2.18 & 1.11 \\
\hline & Others helpful & My teammates contributed to a successful task completion & 1 & 3.39 & 1.17 \\
\hline & Others' observability & I was able to estimate who contributed how much or little & 1 & 4.04 & 1.00 \\
\hline & Observability gainful & Its good that everyone can see who contributes how much & 1 & 3.94 & 1.04 \\
\hline & Freedom during task & I could work the way I wanted to during task processing & 1 & 3.29 & 1.15 \\
\hline \multirow{4}{*}{ 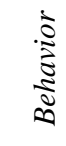 } & Login frequency & Total login times with minimum & & 13.3 & 9.33 \\
\hline & Contribution Forum & Word count of forum contributions per topic block & & 173 & 200 \\
\hline & Contribution Wiki & Word count of wiki contributions per topic block & & 277 & 272 \\
\hline & Participation Equality & Gini coefficient, $0=$ perfectly equal participation & & .74 & .14 \\
\hline
\end{tabular}

\section{RESULTS}

To test the hypothesis H1, we conducted a linear regression $(n=41)$ using the index of equality of user participation in the final group task (day 12 of the second teamwork) as predictor and satisfaction with the group work in this task as outcome variable. Results revealed a statistically significant relationship between the factor equality of participation and group task satisfaction. A significant regression equation was found $F(1,40)=12.30, p=.001$, with an $R^{2}$ of .235 . A strong negative correlation could be detected $(b=-.49$, $S E=1.05, p=.001)$. However, the regression analysis was not significant regarding the first teamwork block. Equality of participation $(0=$ perfectly equal $)$ increased the participants' satisfaction in the second task. Hypothesis $\mathrm{H} 1$ is partly supported. 
To test the hypotheses $\mathrm{H} 2$ and $\mathrm{H} 3$, multivariate analyses of variance (MANOVA) were computed with the vicarious punishment as a fixed factor and the corresponding dependent variables (see Table 2).

Table 2. Results

\begin{tabular}{|c|c|c|c|c|c|c|c|c|c|c|c|}
\hline \multirow[b]{2}{*}{ Variable } & \multicolumn{4}{|c|}{ Vicarious Punishment $(\mathrm{H} 2, \mathrm{H} 3)$} & \multicolumn{4}{|c|}{ VP + Transparency (RQ1, RQ2) } & \multicolumn{3}{|c|}{ Long term (RQ3) } \\
\hline & $F^{(\mathrm{df1}, \mathrm{df} 2)}$ & $p$ & $\eta$ & $M_{\text {none / one }}$ & $F^{(\mathrm{df1} 1 \mathrm{df2})}$ & $p$ & $\eta$ & $M_{\text {low } / \text { high }}$ & $F^{(\mathrm{dfl}, \mathrm{df} 2)}$ & $p$ & $\eta$ \\
\hline constructive mean & $5.25^{(1,36)}$ & .028 & .13 & $3.17 / 3.73$ & & & & & & & \\
\hline equal participation $\mathrm{d}_{12}, \mathrm{t}_{1} 2$ & $22.69^{(1,46)}$ & $<.001$ & .33 & $.645 / .807$ & & & & & & & \\
\hline others' observability $t_{2}$ & $5.03^{(1,27)}$ & .033 & .16 & $4.73 / 4.28$ & $5.08^{(1,26)}$ & .033 & .16 & $4.28 / 3.46$ & & & \\
\hline comprehensive $t_{1}, 1$ item & & & & & $7.49^{(1,31)}$ & .01 & .20 & $3.70 / 4.39$ & & & \\
\hline reassured mean & & & & & $7.51^{(1,33)}$ & .01 & .19 & $4.00 / 2.85$ & & & \\
\hline hints noticed $t_{2}$ & & & & & $6.09^{(1,26)}$ & .021 & .19 & $5.64 / 3.29$ & & & \\
\hline equal participation $d_{12}, t_{-}$ & & & & & $15 \cdot 21^{(1,37)}$ & $<.001$ & .29 & $.747 / .898$ & & & \\
\hline fear $t_{2}$ & & & & & $7.11^{(1,26)}$ & .013 & .22 & $1.86 / 2.73$ & & & \\
\hline transparency $x$ fear $t_{1} \Rightarrow t_{2}$ & & & & & & & & & $6.32^{(1,24)}$ & .019 & .21 \\
\hline transparency $\mathrm{x}$ inactive im & nitation $t_{1}$ & & & & & & & & $4.25^{(1,24)}$ & .05 & .15 \\
\hline transparency $x$ stress $t_{1} \Rightarrow t$ & & & & & & & & & $3.29^{(1,24)}$ & .082 & .12 \\
\hline transparency x equal partic & icipation $d_{1}$ & $2 \mathrm{t}_{1} \Rightarrow \mathrm{t}_{2}$ & & & & & & & $14.11^{(1,37)}$ & .001 & .28 \\
\hline vicarious punishment $\mathrm{x}$ eq & qual partici & pation & $\mathrm{d} 12 \mathrm{t}$ & $\Rightarrow t_{2}$ & & & & & $21.78^{(1,38)}$ & .001 & .36 \\
\hline vicarious punishment $\mathrm{x}$ str & tress $t_{1} \Rightarrow t_{2}$ & & & & & & & & $4.73^{(1,27)}$ & .039 & .15 \\
\hline vicarious punishment $\mathrm{x}$ ob & bjectivity $t_{1}$ & $\Rightarrow t_{2}$ & & & & & & & $3.51^{(1,27)}$ & .072 & .12 \\
\hline
\end{tabular}

$\mathbf{t}_{1 \text { or } 2}=\operatorname{after} 1^{\text {st }}$ or $2^{\text {nd }}$ teamtask; mean $=$ mean across $t_{1}$ and $t_{2} ; \mathbf{d}_{12}=$ day 12 of 14 in a teamtask; VP $=$ Vicarious Punishment

Regarding H2, vicarious punishment had no significant effects on the subjective user perception of inactive teammates (2.1) and satisfaction with group work (2.3), but it influenced, contradicting to our assumption, the observability of others' contribution (2.2) negatively (for an overview see Figure 2). This means that group awareness was higher in the condition without vicarious punishment. Regarding objective user behavior, vicarious punishment had no significant effects on login frequency (2.4), contribution quantity in forum (2.5) and wiki (2.6). However, it influenced equality of participation (2.7), differently than hypothesized, as the participation was more equal in the condition without vicarious punishment (Table 2). Thus, H2.1, H2.3, H2.4, $\mathrm{H} 2.5$, H2.6 are not supported, while $\mathrm{H} 2.2$ and $\mathrm{H} 2.7$ yielded significant differences between vicarious punishment conditions, which are contrary to our assumptions.

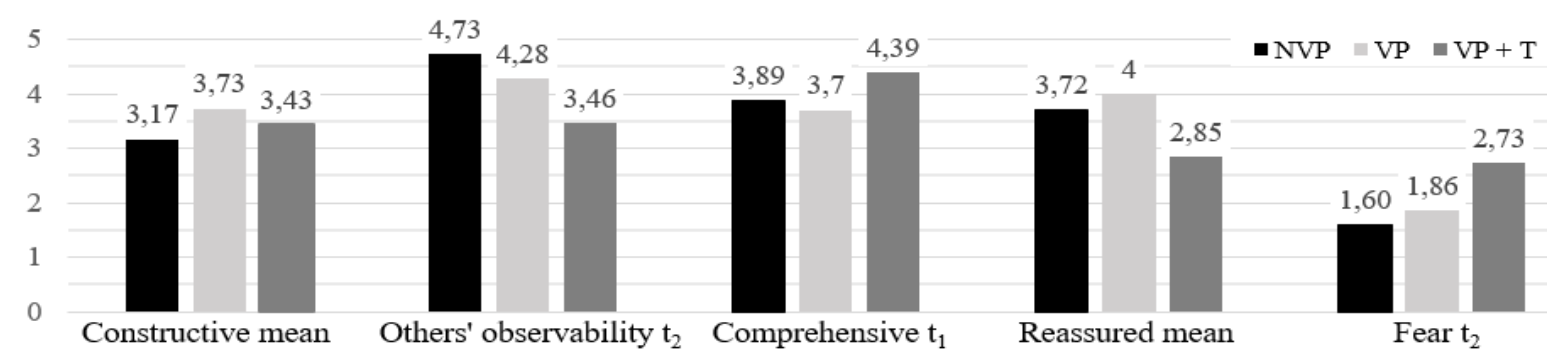

Figure 2. Differences between conditions vicarious punishment (VP), transparency (VP + T) and none (NVP)

Regarding H3 and the perception of the system and the prompting messages, vicarious punishment had no effect on the perception of the system (3.1), but, contrary to our assumption, a significant positive effect on the perception of the prompt messages (3.2) - i.e., they were perceived as more constructive in the vicarious punishment condition (Table 2 \& Figure 2). Thus, H3.1 and H3.2 are not supported, whereby H3.2 showed positive instead of negative effects of vicarious punishment on the perceived constructiveness of the prompt messages.

Further MANOVA analyses were computed to explore research questions RQ1 and RQ2, with transparency of the system (transparency) as a fixed factor and as the corresponding dependent variables (Table 2). Regarding RQ1, transparency had no significant effects on the satisfaction with teamwork (RQ1.4). However, there were significant effects on perception of the system (RQ1.1), the prompt messages (RQ1.2), and the group awareness (RQ1.3). When participants knew more about the functionality of the system, i.e. in the high transparency condition, they perceived it less reassuring that action is taken against inactive members and more afraid to get publicly criticized, too (Figure 2). In this condition with high transparency participants perceived the prompt messages as more comprehensive (Figure 2), but reported to have noticed a lower number 
of prompt messages. However, transparency showed a negative effect on group awareness as observability of others' participation was lower (Table 2). Regarding influence of transparency on users' behavior (RQ2), there was a significant negative effect on equality of participation - it was less equal in the high transparent condition.

To explore differences between first and second group task depending on vicarious punishment and transparency (RQ3), two-factorial repeated measures ANOVAs were conducted with repeated measure on one factor to compare the effects of task participation repetition for each dependent variable among conditions (as a non-repeated between subject factor). Due to the sample size and its potential risk for the function of the Mauchly's test, multivariate tests are reported. Main differences between the conditions have been reported in the prior result section and are disregarded with the smaller sample of the repeated measure analysis.

Transparency showed a significant difference over time (first vs. second teamwork) on stress, fear to also get publicly criticized and the tendency to imitate inactive teammates, as well as on equality of participation. When people knew more about the functioning of the system, participants' stress was higher in the first task and lower in the second task, while in the condition where they knew less, stress was lower in the first group work but higher during the second group work. Similarly, fear to get criticized was higher in the second group task in the transparency condition, while it was lower in the second group task compared to the first group task in the non-transparent condition. However, reported tendency to imitate the behavior of the inactive teammates was lower in the first task and increased when knowing more about the system, while in the low transparency condition, imitation tendency was lower in the second task (see Table 2 \& Figure 3 ).
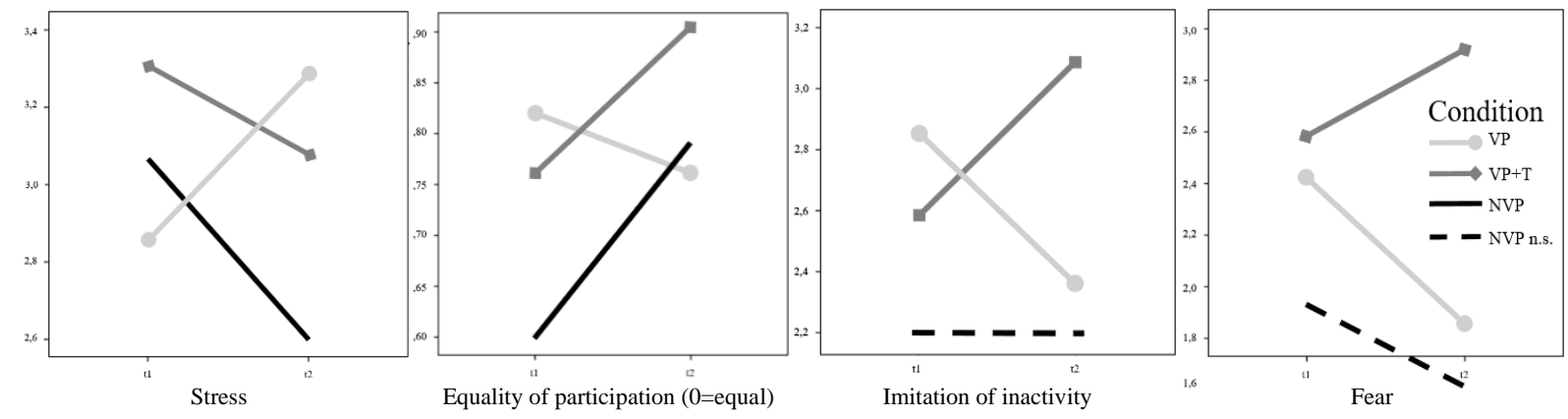

Figure 3. Effects over time (after first/second teamwork): vicarious punishment (VP), transparency (VPT) or none (NVP)

Vicarious punishment showed a significantly different impact over time on stress and a marginally significant effect on perceived objectivity of the prompt messages (see Table $2 \&$ Figure 3 ). Without vicarious punishment participants' stress was already low after the first task but was even lower after the second task, while in the condition with vicarious punishment, stress was initially lower and increasing over time.

Both transparency and vicarious punishment showed a significantly different impact on equality of participation over time (see Table $2 \&$ Figure 3 ). Two days before the deadline, participation was getting more equal over time (first vs. second topic block) in the vicarious punishment condition, while without it, it was getting less equal. Regarding transparency, equality of participation increased over time in the low transparent condition, but it decreased in the high transparent condition.

In order to examine potentially influencing factors of users' objective behavior (RQ4), Pearson correlations with the mean values among the first and the second task were conducted. We analyzed the relationship of subjective data perception of prompts and inner group stress, conflict, and trust, on satisfaction, fear and, lastly yet importantly, on the objective behavior such as forum and wiki contribution amount, and login frequency. Results show several significant relationships among them.

Regarding user's behavior (RQ4.1), for instance, login frequency correlates significantly negatively with fear but positively with both kind of contributions - in forum and wiki, indicating a link between more frequent logins, more contribution and less fear. Furthermore, for the contribution in forum and wiki a significant negative correlation was found with hostility of prompts. Thus, there is a link between the higher amount of contribution and the perception of prompts as less hostile (see Table 3 for a values overview). For research question 4.1 we can conclude that higher login frequency relates to higher amount of contribution and less fear.

Regarding subjective data (RQ4.2 and 4.3), several relevant factors were found. Fear correlates negatively with the perceived objectivity of prompts, but positively with their faultiness ( $p=.054$, marginally significant) (Table 3), indicating a link between higher fear to get publicly criticized and the perception of prompts as less objective and more faulty. Perceived in-group conflict also correlates significantly positively with the perception of prompts faultiness, i.e. the faultier the prompts, the more conflict was perceived. Satisfaction 
with teamwork correlates significantly positively with others' helpful contribution and negatively with coordination stress, in-group conflict and the perception of inactive members as disliked peers, disliked compensation of their inactivity and the tendency to imitate them. This indicates a relationship between higher satisfaction with teamwork and more helpful teammates' contributions, as well as relationships between lower satisfaction and conflict, coordination stress, disliked inactive members, the compensation of their inactivity and lower tendency to imitate inactivity. Similarly, in-group trust correlates significantly positively with satisfaction and helpful contributions of other teammates, but significantly negatively with coordination stress and disliking of inactive teammates. The tendency to imitate inactivity correlates significantly positively with faultiness of prompts but negatively with the objectiveness of prompts (see table 3 ).

Thus, regarding subjective in-group experiences (RQ4.2), we can conclude that the perception of prompts as faultier and less objective relates to the perception of more fear to get publicly criticized. Higher prompts' faultiness is also linked to higher conflict perception. In conclusion regarding perceived fear and satisfaction (RQ4.3), the higher tendency to imitate inactive teammates relates to prompts' higher faultiness and lower objectivity. The higher imitation tendency is also linked to lower satisfaction and more fear. Higher perceived fear relates additionally to less observability of others' contributions, less disliking of inactive teammates and the compensation of their inactivity.

Table 3. Bivariate Pearson's correlations among potentially relevant subjective data and objective behavior

\begin{tabular}{|c|c|c|c|c|c|c|c|}
\hline & Faultiness & Satisfaction & $\begin{array}{c}\text { In-group } \\
\text { trust }\end{array}$ & $\begin{array}{l}\text { Objective } \\
\text { prompts }\end{array}$ & Fear & $\operatorname{Login}^{F}$ & $\begin{array}{l}\text { Forum+Wiki } \\
\text { contribution }\end{array}$ \\
\hline Faultiness & 1 & & -.038 & $-.856^{* *}$ & .264 & -.133 & -.106 \\
\hline Satisfaction & .029 & 1 & $.665^{* *}$ & .041 & .139 & -.048 & -.179 \\
\hline Fact-based & $-.569 * *$ & -.091 & .019 & $.505^{* *}$ & -.151 & .154 & .187 \\
\hline Others' observability & $-.500^{* *}$ & -.024 & -.002 & $.484 * *$ & $-.425^{* *}$ & .163 & .120 \\
\hline Conflict & $.384^{* *}$ & $-.287 *$ & $-.294^{*}$ & $-.307^{*}$ & .083 & -.061 & -.109 \\
\hline Disliked peers & .018 & $-.517^{* *}$ & $-.356^{* *}$ & .032 & $-.280 *$ & .251 & .103 \\
\hline Disliked compensation & n . .032 & $-.521^{* *}$ & $-.359 * *$ & .098 & $-.330 *$ & .238 & .066 \\
\hline Imitation tendency & $.444^{* *}$ & $-.394^{* *}$ & -.195 & $-.489 * *$ & $.360 * *$ & .006 & -.206 \\
\hline Others helpful & -.147 & $.603^{* *}$ & $.577^{* *}$ & .113 & .018 & .068 & .117 \\
\hline Coordination Stress & .026 & $-.526^{* *}$ & $-.335^{*}$ & -.023 & -.132 & .127 & .271 \\
\hline Motivating prompts & $-.276^{*}$ & .163 & .160 & $.523^{* *}$ & -.197 & .175 & .077 \\
\hline Hostile prompts & $.446^{* *}$ & -.200 & -.116 & $-.553^{* *}$ & .248 & -.126 & $-.344^{*}$ \\
\hline Fear & .264 & .139 & .074 & $-.300 *$ & 1 & & \\
\hline Login frequency & -.133 & -.048 & -.026 & .125 & $-.363^{* *}$ & 1 & \\
\hline Forum+Wiki contribut. & t. -.106 & -.179 & -.044 & .259 & $-.430^{* *}$ & $.563^{* *}$ & 1 \\
\hline
\end{tabular}

Regarding the prediction of contribution amount through vicarious punishment (RQ5), fear and login frequency were explored as potential mediators. It was of additional interest whether fear predicts login frequency or vice versa, and whether their perception mediates the effect of vicarious punishment on the amount of contribution. To address this question two double mediation analyses using PROCESS macro for SPSS (Hayes, 2013) were conducted, applying the mean values among both tasks (see Figure 4).

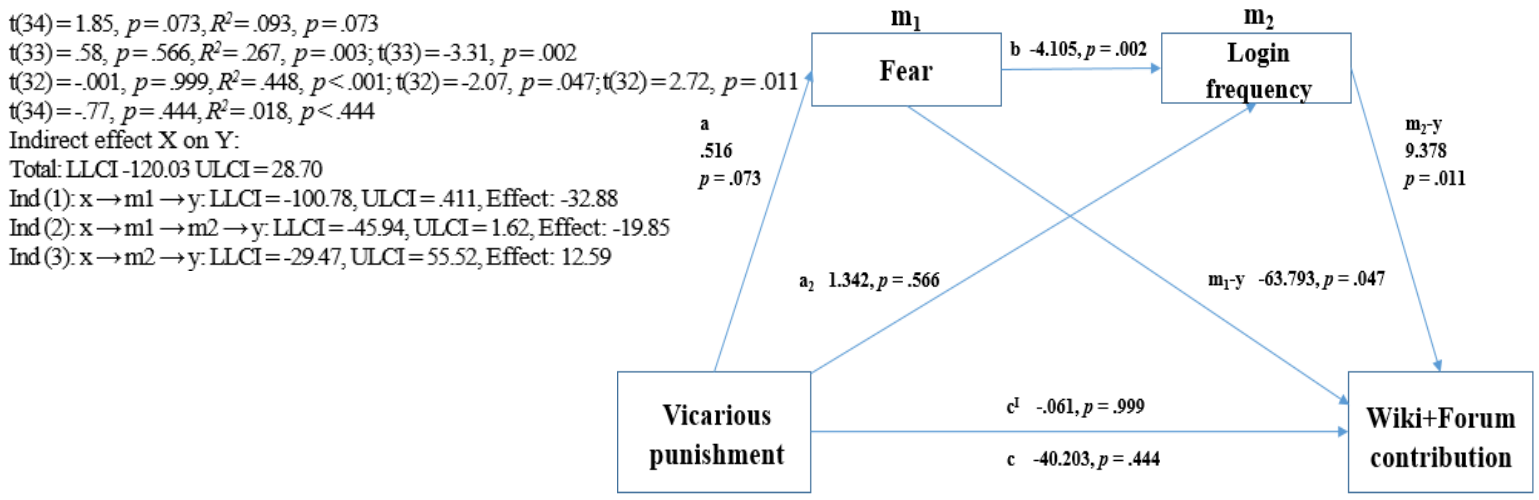

Figure 4. Double mediation models of vicarious punishment predicting contribution, fear and login frequency (RQ5) 
The model, which included fear followed by login frequency, revealed that vicarious punishment was a marginal significant predictor of fear, $b=.52, S E=.28, p<.073$, but not of login frequency, $b=1.34$, $S E=2.31, p=.566$. Further, fear was a statistically significant predictor of contribution, $b=-63.79$, $S E=30.88, p=.047$ and of login frequency, $b=-4.11, S E=1.24, p=.002$. Additionally, login frequency was a predictor of contribution, $b=9.38, S E=3.45, p=.011$. Results showed that vicarious punishment was not a significant predictor of contribution with both mediators, $b=-.06, S E=42.70, p=.999$ and without them, $b=-40.20, S E=51.94, p=.442$, and the indirect effect of $\mathrm{x}$ on $\mathrm{y}$ was not significant (LLCI $=-120.33$, ULCI =28.70, Effect: -40.14$)$. The double mediation model and detailed values can be seen in Figure 4.

\section{DISCUSSION}

We focus on vicarious punishment and transparency of the system in online learning groups, improvement of users' behavior and perception of system, prompts and teamwork. The combined behavioral and survey data in a field experiment indicated equality of participation to predict teamwork satisfaction. Vicarious punishment increased constructiveness of the prompts, but decreased observability of others, i.e. how well students were able to estimate who participated how much. Participation equality was partly affected but no effect on the amount emerged, which is in contrast to earlier findings detecting higher productivity after offline vicarious punishment (Schnake, 1987). With system transparency, prompts were more comprehensive but noticed less frequently, while participants were more afraid to get criticized themselves, found action against inactive teammates less reassuring and others' contribution less observable. Over time (first vs. second teamwork) participation was getting rather more equal with vicarious punishment, but less equal distributed without it, and even more inequal with transparency. Over time stress increased only with vicarious punishment, while it decreased with a transparent system or without punishment. Transparency increased the fear to get criticized over time but also the tendency to imitate inactivity, while both decreased without transparency. These results are partly in line with the recommendations for balanced transparency of the system (Kizilcec, 2016).

Vicarious punishment showed some beneficial long term effects and will need to be investigated in large settings since our sample had to combat high drop-out rates. Partly, the effects might be related to the transparency paradox (Bernstein, 2012), which was not a subject of the present study. According to the transparency paradox, observability counterintuitively reduced workers' performance, possibly because it motivated to hide activities (applying time and costs consuming means).

Regarding potentially relevant factors which influence the objective behavior, several significant correlations emerged. The more frequent logins, the more contributions and the less fear was perceived by the students. The link between participation and contribution is in line with Chavez and Romero (2013), who indicated low level of participation as a main difficulty in CSCL, and participation as highly important and increasing group productivity, perception and results of learning, as well as the evaluation of results' quality and satisfaction. Additionally, the more contributions, the less hostile users' perception of prompts was. This is potentially linked to the own invested time and effort, i.e. the more students participated themselves, the worse and more unfair they might perceive others' inactivity, and therefore perceive prompts highlighting them indeed not as significantly "more legitimate", but as less hostile.

Regarding subjective user perception of prompts and in-group experiences, the less fear, the more objective and less faulty prompts were perceived. Students might feel secure and invulnerable regarding vicarious punishment, if they do contribute enough. However, in case they do not trust the system and the prompts, as well as the algorithms behind the vicarious punishment procedure, they might still be afraid of faulty and less objective choices of the system. Perceived faultiness of prompts was linked to less observability of others' contribution and trust in the fact-based character of the system. The existence of faulty prompts and a system, which students do not trust to be fact-based, could decrease the perceived possibility to estimate how much others have contributed. Further, with higher perception of faultiness, prompts were also perceived as more hostile, which indicates the importance of the system's accuracy. This relationship might be discussed as a potential hint to the existence of systems' idiosyncrasy credit (Hollander, 1958). As a kind of legitimacy or the right to act contrary to norms after following them sufficiently before, having earned enough idiosyncrasy credit allows to do so without negative consequences. In case of faultiness, system hints are perceived as more hostile since they are incorrectly delivered. Certain characteristics could replace the system's positive "proficiency", signal expertise and credibility, and finally enhance the system's legitimacy to highlight inactive team members. The opposite, kind of discredit, is expected if prompts are perceived faulty, as system's negative 
proficiency. Furthermore, reported higher tendency to imitate other inactive teammates were perceived with higher perception of faultiness. This is a potential indicator of discrediting the system. If students do not trust the system's correct functions, they might also be less motivated to stop inactivity, perceiving the whole system as not functioning well and not threatening for them.

Further, regarding satisfaction, fear and the tendency to imitate inactivity, the more satisfaction, the more people trusted their teammates. Trust and satisfaction, however, increased with the perception of others' contributions as helpful for the group success, and with the perception of less conflict, less coordination stress, less inactivity dislike (of inactive peers and of the need to compensate them) as well as lower tendency to imitate inactivity. Furthermore, as stated above, we also found that equality of participation predicts satisfaction. Nevertheless, not only the mere presence of inactivity in groups, as a common challenge for collaboration, but also the resulting prompts addressing teammates to take up work - both potentially foster conflict and disliking. Taken together, these findings underline even more the need for automated mediation and alternative prompting solutions. These measures can be promising in order to gain the benefits of prompts and group conflicts without the costs of conflicts and personal disliking. Negative consequences in the sense of vicarious punishment are just one of many options needing further investigations in this field.

Yet, it should be acknowledged that these relationships can be interpreted in both directions since correlations do not allow us to interpret any directions. Thus, are conflict and disliking in e-learning groups contra-productive for satisfaction and trust or vice versa? Regarding the negative correlation of login frequency and fear, it stays unclear whether participants had more fear to also get criticized publicly the less frequently they logged in or the other way around. Mediation models' results showed non-significant total, direct and indirect effects, which indicate that it is indeed not easy to detect causal relationships in this area. However, fear significantly negatively predicted contribution as well as login frequency. Additionally, login frequency positively predicted the contribution amount. We can conclude that participants' fear predicted their less frequent logins and less contributions, although more frequent logins predict more contributions. Future work should optimize and customize vicarious punishment to be sufficiently threatening but also sufficiently transparent, without inducing feelings of surveillance. Such balanced prompts could be more easily accepted, without leading to login and contribution avoidance. Thus, studies could focus on the inclusion of mechanisms to improve vicarious punishment as severe and mild simultaneously, and to avoid the transparency paradox when informing about system's functioning. Similarly, the negative correlation of fear and contribution, as well as contribution and hints noticed remain to be clarified by future research.

This study has several limitations that should be acknowledged. First, a not-fully-crossed design was applied. The experimental group with vicarious punishment and low transparency was included in both comparisons. In case of specific characteristics or failures, these could be implied in all study results. An experimental group without transparency included should be applied in future research, as well as a control group without any prompts. Second, equality of participation was measured as a group value, further decreasing sample size. Therefore, a higher number of participants in general, as well as a higher number of participating groups should be tested in future studies. Third, according to Collazos et al. (2014), collaboration does not happen automatically by building a team and letting it solve a task. Therefore, tasks were composed, supervised by pedagogical experts, as requiring collaboration but deliberately allowing success, also with less teammates if necessary in case of dropout. Thus, we did not measure collaboration quality, but this is recommended for future work. However, in a manipulation check regarding noticed prompts, participants reported to have noticed them frequently. This measure should be considered cautiously since the phenomenon of banner blindness proposes that salient stimuli are often missed by users (Sun, Lim \& Peng, 2013). Future studies should take alternatives into account. Further, the questionnaire was filled in twice - after each team task. Future long-term tests should be applied, including a measurement of stress, fear etc. also before the experimental manipulation. A better balance between short questionnaires in order to avoid dropout and longer standardized questionnaires is needed. Lastly, effect sizes of the results were small to medium.

In conclusion, participation equality predicts satisfaction. Vicarious punishment revealed more constructive prompts, but less observability of others, i.e. the ability to estimate others' contribution, which was even lower when a detailed system function description was included for higher transparency. However, transparency revealed participants to be more afraid, and a negative correlation with number of contributions was shown. However, it remains unclear whether less fear is related to more contributions or less contribution to more fear. Regarding the carrots and sticks procedure, therefore further work is needed. Transparency might help have them appear as beneficial and credible as possible, especially in case carrots do not provide sufficient motivation, so that indeed sticks and other mediators are needed. 


\section{ACKNOWLEDGEMENT}

This study is based on data collected in the "IKARION"-Project funded by the BMBF (16DHL1013). We thank Marcel Finkel for the assistance and all our collaborators for their contributions.

\section{REFERENCES}

Bernstein, E. S. (2012). The transparency paradox: A role for privacy in organizational learning and operational control. Administrative Science Quarterly, 57(2), 181-216. https://doi.org/10.1177/0001839212453028

Chavez, J., \& Romero, M. (2012). 'Group awareness, learning and participation in computer supported collaborative learning (CSCL)'. Procedia - Social and Behavioral Sciences. 46, 3068-3073

Collazos, C.A., et al. (2014) 'Design guidelines to foster cooperation in digital environments', Technology, Pedagogy and Education, Vol. 23, No. 3, pp.375-396. doi:10.1080/1475939X.2014.943277.

Erdmann, J., et al. (2017). Challenges in implementing small group collaboration in large online courses. In B. K. Smith, B. K., M. Borge, E. Mercier, \& K. Y. Lim (Eds.), Making a difference: Prioritizing equity and access in CSCL, 12th International Conference on Computer Supported Collaborative Learning (CSCL) 2017, Volume 2. Philadelphia, PA: International Society of the Learning Sciences.

Hayes, A. F. (2013). Introduction to mediation, moderation, and conditional process analysis: A regression-based approach. New York, NY: Guilford.

Hollander, E. P. (1958). Conformity, status, and idiosyncrasy credit. Psychological Review, 65(2), $117-127$. http://dx.doi.org/10.1037/h0042501

Kim, T., \& Hinds, P. (2006). Who Should I Blame? Effects of Autonomy and Transparency on Attributions in Human-Robot Interaction. In 15th IEEE International Symposium on Robot and Human Interactive Communication (Ro-MAN) (pp. 80-85). IEEE.

Kizilcec, R. F. (2016, May). How much information? Effects of transparency on trust in an algorithmic interface. In Proceedings of the 2016 CHI Conference on Human Factors in Computing Systems (pp. 2390-2395). ACM.

Kyewski, E., \& Krämer, N. C. (2018). To gamify or not to gamify? An experimental field study of the influence of badges on motivation, activity, and performance in an online learning course. Computers \& Education, 118, 25-37. doi:10.1016/j.compedu.2017.11.006

Malouff, J., Thorsteinsson, E., Schutte, N., \& Rooke, S. E. (2009). Effects of vicarious punishment: A meta-analysis. The Journal of General Psychology, 136(3), 271-286. https://doi.org/10.3200/GENP.136.3.271-286

Nussbaumer P., \& Matter I. (2011). What you see is what you (can) get? Designing for process transparency in financial advisory encounters. In P. Campos, N. Graham, J. Jorge, N. Nunes, P. Palanque, \& M. Winckler (Eds.), IFIP Conference on Human-Computer Interaction (pp. 592-603). Berlin, Heidelberg, DE: Springer. https://doi.org/10.1007/978-3-642-23774-4_24

O'Fallon, M. J., \& Butterfield, K. D. (2012). The influence of unethical peer behavior on observers' unethical behavior: A social cognitive perspective. Journal of Business Ethics, 109(2), 117-131.https://doi.org/10.1007/s10551-011-11117

Schnake, M. E. (1987). Vicarious punishment in a work setting: A failure to replicate. Psychological Reports, 61(2), 379-386. https://doi.org/10.2466/pr0.1987.61.2.379

Sinha, R., \& Swearingen, K. (2002). The role of transparency in recommender systems. In Proceedings of the CHI'O2 Conference on Human Factors in Computing Systems: Changing the world, changing ourselves, Minneapolis (pp. 830-831). https://doi.org/10.1145/506443.506619

Strauß, S., Rummel, N., Stoyanova, F., \& Krämer, N. (2018). Developing a library of typical problems for collaborative learning in online courses. In J. Kay \& R. Luckin (Eds.) (2018), Rethinking Learning in the Digital Age: Making the Learning Sciences Count, 13th International Conference of the Learning Sciences (ICLS) 2018, Volume 2 (pp. 1045-1048). London, UK: International Society of the Learning Sciences.

Stoyanova, F., \& Krämer, N. (2019). Please don't shoot the messenger! Prompts in online learning groups - Influences of nudging messages' sender and publicness on recipients' perception and attribution. In K. Lund, G. Niccolai, E. Lavoué, C. Hmelo-Silver, G. Gweon, \& M. Baker (Eds.), A Wide Lens: Combining Embodied, Enactive, Extended, and Embedded Learning in Collaborative Settings: 13th International Conference on Computer Supported Collaborative Learning (CSCL) 2019 (pp. 256-263). Lyon, France: International Society of the Learning Sciences.

Sun, Y., Lim, K. H., \& Peng, J. Z. (2013). Solving the distinctiveness-blindness debate: A unified model for understanding banner processing. Journal of the Association for Information Systems, 14(2), 49-71.

*Questionnaires overview at OSF: https://mfr.osf.io/render?url=https\%3A\%2F\%2Fosf.io\%2Fktcp5\%2Fdownload 